\title{
What is a non-truth-functional logic?
}

\author{
João Marcos \\ DIMAp / CCET, UFRN, Brazil \\ jmarcos@dimap.ufrn.br
}

\begin{abstract}
What is the fundamental insight behind truth-functionality? When is a logic interpretable by way of a truth-functional semantics? To address such questions in a satisfactory way, a formal definition of truthfunctionality from the point of view of abstract logics is clearly called for. As a matter of fact, such a definition has been available at least since the 70 s, though to this day it still remains not very widely well-known.

A clear distinction can be drawn between logics characterizable through: (1) genuinely finite-valued truth-tabular semantics; (2) no finite-valued but only an infinite-valued truth-tabular semantics; (3) no truth-tabular semantics at all. Any of those logics, however, can in principle be characterized through non-truth-functional valuation semantics, at least as soon as their associated consequence relations respect the usual tarskian postulates. So, paradoxical as that might seem at first, it turns out that truthfunctional logics may be adequately characterized by non-truth-functional semantics. Now, what feature of a given logic would guarantee it to dwell in class (1) or in class (2), irrespective of its circumstantial semantic characterization?

The present contribution will recall and examine the basic definitions, presuppositions and results concerning truth-functionality of logics, and exhibit examples of logics indigenous to each of the aforementioned classes. Some problems pertaining to those definitions and to some of their conceivable generalizations will also be touched upon.
\end{abstract}

Keywords. Abstract logics, formal semantics, truth-functionality.

\section{What does a truth-functional logic look like?}

"That is another of your odd notions," said the Prefect, who had the fashion of calling everything 'odd' that was beyond his comprehension, and thus lived amid an absolute legion of 'oddities'.

-Edgar Allan Poe, The Purloined Letter, 1845.

Traditionally, when one talks about a truth-functional (propositional) logic, the picture that one has in mind is that of a logic characterized by truth-tables. In 
such a logic, the Principle of Compositionality, according to which the meaning of a complex expression is determined by the meanings of its constituent expressions and the rules used to combine them, is implemented in a straightforward way: Every sentence $\varphi$ having an $m$-ary symbol (c) as its main connective applied to a collection of $m$ immediate subsentences should be interpreted by some sort of similar $m$-ary operator applied to the interpretation of each of the immediate subsentences.

The paradigmatic case of a truth-functional logic, of course, is that of classical logic. The full language of classical propositional logic, in one version or another, contains a collection of $m$-ary connectives that construct complex sentences that may be characterized by 2 -valued truth-tables with (at most) $2^{m}$ rows, each row representing a possible truth-value assignment to its component sentences (in fact, representing only a partial assignment, or a class of assignments that coincide with the values in that row when the domain of such assignments is restricted to the $m$ atoms that are represented in the table). So, a truth-table for a binary connective (c) might look like in Fig. 1, where each $w_{i j}$ represents a truth-value in $\{0,1\}$. Each row of the truth-table represents a 'state of affairs' - if the value $w_{i j}$ assigned to the sentence $\alpha \subset \beta$ is 0 in a given state of affairs, this sentence is said to be 'false', otherwise it is said to be 'true'.

\begin{tabular}{c|c|c}
$\alpha$ & $\beta$ & $\alpha \subset \beta$ \\
\hline 0 & 0 & $w_{00}$ \\
\hline 0 & 1 & $w_{01}$ \\
\hline 1 & 0 & $w_{10}$ \\
\hline 1 & 1 & $w_{11}$
\end{tabular}

Figure 1: Truth-table for the binary connective (c)

Another usual way of representing a truth-table for an $m$-ary connective is by way of an $m$-dimensional square array, as in Fig. 2.

\begin{tabular}{|c|c|c|}
\hline C & 0 & 1 \\
\hline 0 & $w_{00}$ & $w_{01}$ \\
\hline 1 & $w_{10}$ & $w_{11}$ \\
\hline
\end{tabular}

Figure 2: Matrix-like representation for the binary connective (C)

One further natural idea, in calculating states of affairs and their effects over complex sentences, is to consider a set of basic bricks, 'atomic sentences' that are, in principle, free to be assigned any truth-value among those values that we have at our disposal.

Now, how could the above notion of truth-tables be generalized for an arbitrary number of 'truth-values'? The conventional way of doing this builds on the idea of considering many-valued logics containing many 'degrees of falsehood and of truth(hood)', instead of a less nuanced 2-valued logic, as above. Now the set of truth-values may be, say, any collection $\{0, \ldots, x, y, \ldots, 1\}$, where the 
'undesignated values' $\{0, \ldots, x\}$ allow for shades of falsehood, and the 'designated values' $\{y, \ldots, 1\}$ allow for shades of truth. In that case, a truth-table for a binary connective (c) could then look like in Fig. 3.

\begin{tabular}{|c|c|c|c|c|c|c|}
\hline C & 0 & $\ldots$ & $x$ & $y$ & $\ldots$ & 1 \\
\hline 0 & $w_{00}$ & $\ldots$ & $w_{0 x}$ & $w_{0 y}$ & $\ldots$ & $w_{01}$ \\
\hline$\vdots$ & $\vdots$ & $\ddots$ & $\ldots$ & $\ldots$ &. & $\vdots$ \\
\hline$x$ & $w_{x 0}$ & $\ldots$ & $w_{x x}$ & $w_{x y}$ & $\ldots$ & $w_{x 1}$ \\
\hline$y$ & $w_{y 0}$ & $\ldots$ & $w_{y x}$ & $w_{y y}$ & $\ldots$ & $w_{y 1}$ \\
\hline$\vdots$ & $\vdots$ &. & $\ldots$ & $\ldots$ & $\ddots$ & $\vdots$ \\
\hline 1 & $w_{10}$ & $\ldots$ & $w_{1 x}$ & $w_{1 y}$ & $\ldots$ & $w_{11}$ \\
\hline
\end{tabular}

Figure 3: A many-valued binary matrix

The above generalization procedure might seem intuitive and the figures might seem suggestive, but there are a lot of hidden presuppositions about the structure of sentences and about the nature of many-valued interpretations which had better be spelled out here in more detail.

Let's start by syntax, and fix from this point on a set At of atoms. Let Cnt $=\left\{\mathrm{Cnt}_{n}\right\}_{n \in \mathbb{N}}$ be a family of logical constants, where each $\mathrm{Cnt}_{m}$ is a set of $m$-ary connectives. The signature $\Sigma$ of a language defined by the previous elements will be given by the collection $\bigcup$ Cnt. As usual, the set $\mathcal{S}$ of sentences that can be written in the above language will be constructed as the free algebra (recursively) generated over At with respect to $\Sigma$. So, if $p \in$ At, then $p \in \mathcal{S}$, and if $\left\{\alpha_{k}\right\}_{k<m} \subset \mathcal{S}$ and (c) $\in \mathrm{Cnt}_{m}$, then (C) $\left(\alpha_{0}, \ldots, \alpha_{m-1}\right) \in \mathcal{S}$. (Notice that we also allow for the presence of 0 -ary connectives and for the eventuality of empty languages.) Any set of sentences that can be obtained through the above procedure will be said to have an algebraic character.

The trick for associating now a semantics, by way of truth-tables, to the above language and a meaning to its corresponding set of sentences goes more or less like this. For each connective (c) a homonymous 'operator' will be sought that can be represented in the appropriate (functional) way. To interpret a sentence of the form $\alpha \subset \beta$, for instance, one will try to compose interpretations of $\alpha$, of (c), and of $\beta$. For the sake of economy, a minimal such collection of truth-values will be sought to do the job. As we said before, atoms will be allowed to assume any truth-value from that set, as determined by some given 'assignment' mapping that incorporates the intuition about representing a state of affairs. The value assigned by this mapping to the sentence $\alpha \subset \beta$ will be $w_{x y}$, as in Fig. 3, as soon as $x$ is the value assigned to $\alpha$ and $y$ is the value assigned to $\beta$. The interpretation of a given connective will be determined then by the set of all legal 'valuations' that extend the initial assignments, following this exact same pattern. With some luck, this will be representable by way of tables such as the above ones. In that case, the total functional operator associated to (C) will be such that (C) $(x, y)=w_{x y}$. What we are trying to build, of course, is a $\Sigma$-algebra of truth-values that faithfully mirrors the underlying syntax, but that 
now provides what is conventionally called 'semantics', a set of homomorphisms assigning to the sentences of a logic some sort of meaning that escapes their mere syntactical formulation. In theory, this might seem like we are just changing one formalism by another, one algebra by another. In practice, though, if a logic is characterized, say, in proof-theoretical terms, or through some abstract description, axiomatic or not, it is very often a matter of insightful discovery the realization that this same logic can be characterized by a semantics complying with the above standards.

Our exposition this far has tried to remain at a naîve level. It is a bit as if we had some intuitive notion of truth-functionality that we wanted to approach and capture, in a way that will allow us both to be precise about its behavior and at the same time free to vary its determining elements. The following section will, accordingly, make the above notion of truth-functional semantics more precise, step by step. The ultimate task will be that of convincing the reader that truth-functionality is both a delicate and an objective property of a logic, a property that should be approached thus through abstract means, by way of Universal Logic postulates.

As we will see, some logics can be characterized by truth-functional semantics with a finite number of truth-values, and other logics will need an infinite number of truth-values. Nonetheless, many logics from the latter class will still prove to have some sort of adequate finite-valued semantics if we only relax the very conditions defining truth-functionality. However - and this is of utmost importance - some logics simply fail to have a truth-functional semantics at all. Indeed, some logics just cannot have a many-valued semantics with all the characteristics embodied in the above illustrated notion of truth-tables. Again, as we shall see, relaxing the definition of truth-functionality might allow some such logics to be recaptured, while the main advantages of truth-functionality are still retained. The remainder of this paper will clarify which logics belong to each of the above mentioned classes, and point to some open directions of investigation concerning that classificatory issue.

\section{Recipe for truth-functionality}

We may regard the present state of the universe as the effect of its past and the cause of its future. An intellect which at a certain moment would know all forces that set nature in motion, and all positions of all items of which nature is composed, if this intellect were also vast enough to submit these data to analysis, it would embrace in a single formula the movements of the greatest bodies of the universe and those of the tiniest atom; for such an intellect nothing would be uncertain and the future just like the past would be present before its eyes.

-Pierre-Simon, Marquis de Laplace, Essai Philosophique sur les Probabilités, 1814.

This section will entertain a semantic characterization of the phenomenon of truth-functionality in 10 maxims. These will be argued to be the underlying 
elements of the above illustrated 'intuitive' picture of a logic characterized by way of truth-tables.

To be sure that we are talking about a truth-functional logic $\mathcal{L}$ over a set of sentences $\mathcal{S}$, the first thing that will need to be checked is that:

(TF1) $\mathcal{L}$ can be given a many-valued semantics. A valuation over $\mathcal{S}$ is a total mapping $\S: \mathcal{S} \longrightarrow \mathcal{V}_{\S}$ that assigns entities called truth-values to the sentences. A semantics Sem here is just a collection $\left\{\oint_{k}\right\}_{k \in K}$ of valuations. Notice that we do not initially assume anything about the cardinality of $\mathcal{S}$, of $\mathcal{V}_{\S}$, or of Sem.

To fully realize the above maxim, for sure, we had better describe in more detail the counter-domains of the valuation mappings. So, the second thing we need is:

(TF2) There are (two kinds of) truth-values. Each $\mathcal{V}_{\S}$ will in fact be quasipartitioned into designated and undesignated values, respectively denoted by $\mathcal{D}_{\S}$ and $\mathcal{U}_{\S}$. Thus, $\mathcal{D}_{\S} \cup \mathcal{U}_{\S}=\mathcal{V}_{\S}$, and $\mathcal{D}_{\S} \cap \mathcal{U}_{\S}=\varnothing$ (recall that in a quasi-partition a partition class may be empty). We will often commit below an abuse of language by calling any value in $\mathcal{D}_{\S}$ 'true' and any value in $\mathcal{U}_{\S}$ 'false'.

Why do we need truth-values to come in exactly two flavors? Well, here comes into play a (not very well) hidden presupposition of the author of this study: You do not really have a 'logic' until you clarify what is its associated inferential behavior. In other words, logic is not about what is true or what is false; it is about what-follows-from-what. But then, to realize that intention, one might assume that:

(TF3) $\mathcal{L}$ has associated entailment relations. It is nowadays customary to consider different levels of inference. Here, from a semantic perspective, a 'canonical' notion of local entailment $\models_{\S} \subseteq \operatorname{Pow}(\mathcal{S}) \times \mathcal{S}$ associated to a given valuation $\S$, may be defined by setting:

$$
\Gamma=_{\S} \alpha \text { iff } \S(\Gamma) \cap \mathcal{U}_{\S} \neq \varnothing \text { or } \S(\{\alpha\}) \cap \mathcal{D}_{\S} \neq \varnothing .
$$

This means that a conclusion $\alpha$ may be extracted from a set of premises $\Gamma$ according to a valuation $\S$ if either some premise $\gamma \in \Gamma$ is 'false' or if the conclusion $\alpha$ is 'true'. In the present (single-conclusion) framework, this can also be rephrased by requesting the conclusion to be true once all premises are assumed to be true; to put it in a slogan, one might say that "truth is to be preserved from premises to conclusion". Next, the global entailment $\models$ sem $\subseteq$ $\operatorname{Pow}(\mathcal{S}) \times \mathcal{S}$ associated to a semantics Sem is defined by setting:

$$
\Gamma \models_{\text {Sem }} \alpha \text { iff } \Gamma \models_{\S} \alpha \text { for every } \S \in \text { Sem. }
$$

Notice how the above definition of global entailment mentions the local one. Indeed, set-theoretically, we have $=_{\text {Sem }}=\left(\bigcap_{\S \in \text { Sem }} \models_{\S}\right)$.

Remark 1 In an extreme situation, one might take (TF3) as just saying that a logic must come equipped with a family of entailment relations, conveniently interrelated and organized, say, by way of some lattice structure (cf. [5]), providing a sort of pluralist approach to logic. One might also imagine, alternatively, an obvious way of identifying the local and the global entailment, by considering 
logics characterized by what we may call unitary semantics, defined by a single valuation (cf. [29]).

Of course, the above described notion of local entailment, heavily dependent on the neat bivalent partition of truth-values into designated and undesignated values, is not written in the sky. But it is the one notion that seems to be encrusted in Tarski's original concept of 'following logically' (cf. [42]) and that has been extensively followed by the community ever since. It is also the one adopted in the present paper.

Remark 2 Needless to say, deviant notions of entailment are possible, and even notions that are not based on a bivalent partition of the truth-values. Suppose for instance that one starts from a set of truth-values partitioned into designated and undesignated values, as before, but now one separates from the latter a subset of rejected values, $\mathcal{R}_{\S} \subseteq \mathcal{U}_{\S}$. Following $[27,40]$, one may now very naturally consider a new notion of local entailment according to which designated values follow from non-rejected ones (in the spirit of an original idea by Łukasiewicz):

$$
\Gamma \models_{\S} \alpha \text { iff } \S(\Gamma) \cap \mathcal{R}_{\S} \neq \varnothing \text { or } \S(\{\alpha\}) \cap \mathcal{D}_{\S} \neq \varnothing .
$$

The global version of this new inherently trivalent notion of entailment is defined as in (TF3), above. Call this alternative semantic environment $(\mathrm{TF} 3)^{Q}$. The inferential behavior of a logic characterized by $(\mathrm{TF} 3)^{Q}$ needs not coincide with anything that could be defined using the canonical notion of global entailment introduced by (TF3), specially in case 'rejected' does not coincide with 'undesignated'.

It so happens, it should be acknowledged, that the usual definition of truthfunctionality often makes presuppositions about the very structure of the underlying language. At least since [22] the following has been widely assumed to be a rather natural feature of abstract logics:

(TF4) $\mathcal{S}$ has an algebraic character. This means, as we saw in the last section, that the set of sentences is constructed as a free algebra.

The main practical use of the latter maxim, of a purely syntactical flavor, is to allow for meta-linguistic operations to be defined and performed recursively and for properties to be proven by induction on the structure of sentences. An important such operation, from the proof-theoretical viewpoint, is the so-called rule of uniform substitution, according to which atoms of the language can be uniformly exchanged by other (possibly more complex) sentences. It is just a small step then to require inferences to be insensible to uniform substitutions: Whatever can be asserted with the use of sentences having a certain 'logical form' should still be assertible with the use of other sentences sharing that same form. It has in fact often been assumed in the literature that closure under uniform substitution of atoms by complex sentences is presumed by the received (Aristotelian?) notion of 'logical form'. But this needs not be so. For a start, as one can learn from [25], no non-trivial extension of the consequence relation 
associated to classical logic (such as a supra-classical non-monotonic logic) can be defined unless uniform substitution is abandoned. In addition, [37] defends the view that 'the strongest reasonable requirement concerning closure under substitutions' allows only for the relettering of the basic bricks that construct complex sentences, with atoms being uniformly substituted by other atoms, but not in general by arbitrary complex sentences. The latter stricter requirement has indeed a strong semantic motivation: Truth-values that can possibly be assigned to a given sentence should not be allowed to simply disappear after a substitution is performed over it.

Still and all, the regular version of closure under uniform substitutions is an ordinary feature of many logics, and of truth-functional logics in particular. As advanced in the above paragraph, the algebraic character of $\mathcal{S}$ allows for endomorphisms $\varepsilon$ to be defined over $\mathcal{S}$ by simply describing how atoms are mapped into other sentences (atomic or complex), and then by extending this mapping into a unique homomorphism over the whole algebra of sentences: If (C) $(\alpha, \beta)$ is the original sentence, (C) $\left(\alpha^{\varepsilon}, \beta^{\varepsilon}\right)$ is the sentence associated to it by the endomorphism $\varepsilon$. Then, if the inferences of $\mathcal{L}$ are assumed to be closed under such endomorphisms, any particular inference sanctioned by $\mathcal{L}$ that involves sentences of the form $C(\alpha, \beta)$ can be immediately restated as an equally sanctioned inference involving now sentences of the form C $\left(\alpha^{\varepsilon}, \beta^{\varepsilon}\right)$. The semantic effect of this is to make sure that a sentence of a certain form does not allow for more truth-values to be assigned to it as it gets increasingly complex. Let $\operatorname{Sem}[\varphi]=\{\S(\varphi): \S \in \operatorname{Sem}\}$ be the set of all truth-values assumed by a sentence $\varphi$ according to a given semantics. Then, what we have just said amounts to requiring shorter sentences to be, in a sense, at least as semantically 'representative' as longer sentences obtained from the former through the uniform substitution of their atoms by other sentences, that is:

(TF5) The many-valued semantics of $\mathcal{L}$ is representative. This is intended to mean that $\operatorname{Sem}[\varphi] \supseteq \operatorname{Sem}\left[\varphi^{\varepsilon}\right]$, where $\varepsilon$ is an endomorphism $\varepsilon: \mathcal{S} \longrightarrow \mathcal{S}$ that homomorphically extends some substitution $u s:$ At $\longrightarrow \mathcal{S}$.

As remarked above, logics that fail closure under uniform substitution still allow, more often than not, for some sort of restricted representativeness, in which endomorphisms extend some relettering $r s:$ At $\longrightarrow$ At of the atoms. In combination with the next two maxims, such more relaxed logics would very naturally impose, thus, that $\operatorname{Sem}[\varphi]=\operatorname{Sem}\left[\varphi^{\varepsilon}\right]$.

All the above maxims taken together still leave us with a lot of living space. Truth-functional logics, however, are much more strict in the way they assign truth-values to the sentences of a logic. To start with, they also presuppose:

(TF6) The sets $\mathcal{V}, \mathcal{D}$ and $\mathcal{U}$ are fixed, for every $\S \in$ Sem. Each valuation of a given semantics is supposed to deal thus with essentially the same set of truth-values.

Remark 3 In view of Remark 1, even after we fix the set of truth-values $\mathcal{V}$ it might still be natural to consider an $I$-indexed family of quasi-partitions of it into subsets $\mathcal{D}_{i}$ and $\mathcal{U}_{i}$, for $i \in I$. In this case, a natural all-encompassing 
definition of global entailment would originate if we considered a 'bundle' of local consequence relations $\models_{i}$, for $i \in I$, each one associated to a member of the above family of quasi-partitions. Such a definition is already to be found as early as in [44], and lays at the basis of the study of the so-called 'generalized matrices' (cf. [18]). Given a set of sentences $\mathcal{S}$ and an entailment relation $\models$ defined as in (TF3), we call $\Gamma^{\mathbf{c}}=\{\gamma: \Gamma \models \gamma\}$ the right-closure of $\Gamma \subseteq \mathcal{S}$, and say that $\Gamma$ is closed in case $\Gamma=\Gamma^{\mathbf{c}}$. Then, a special case of generalized matrix is provided by the so-called 'Lindenbaum bundle', for which we consider $\mathcal{V}=\mathcal{S}$, and for each closed set of sentences $\Gamma$ we set $\mathcal{D}_{i}=\Gamma$ and consider the unitary semantics given by the identity mapping over the set of sentences / truth-values. Note that, once we do fix, in the above maxim, the sets of designated and undesignated values, $\mathcal{D}$ and $\mathcal{U}$, it is obvious that the interesting examples of Lindenbaum bundles in particular and of generalized matrices in general are excluded from the realm of truth-functional characterizations. Later on (Fact 11) we will see, indeed, that what we are looking for in this section is a single set of characterizing operators for a single given consequence relation.

In addition to the above presuppositions, as it has been advanced in the previous section, the atoms should be freely allowed to assume any truth-value, that is, they stand a priori to the same chance of reflecting any possible state of affairs. So:

(TF7) The set of valuations is laplacian, that is, $\operatorname{Sem}[p]=\mathcal{V}$, for each $p \in$ At. If the language of a logic has an element that looks like an atom, but that assumes only a specific truth-value throughout the semantics, this element should be regarded as a 0-ary connective / operator, instead of a sort of 'distinguished atom' - otherwise, that could mean trouble for the above postulated notion of representativeness. Examples of such phenomenon are provided by the symbols $I, \perp$ and $\top$ that appear in Section 3, below - the particular truthvalue assumed by each of those symbols is always resistant to substitutions.

We now render explicit the assumption that, except for the exchange of truth-values for atoms, the language mentioned the semantic characterization has the same structure as the one assumed by the purely syntactical considerations in (TF4). In fact:

(TF8) There is a set of operators $O p=\left\{O p_{n}\right\}_{n \in \mathbb{N}}$ over $\mathcal{V}$ of the same similarity type as the set of connectives Cnt. Accordingly, Op and Cnt will share the same propositional signature. I systematically commit here an abuse of notation and use the same symbol to denote both a connective (syntax) and its associated operator (semantics), assuming that the context, in each case, will always help us to tell which of these we are talking about.

The two remaining maxims will show how the above operators should be interpreted and how a $\Sigma$-algebra can be defined on $\mathcal{V}$ that is homomorphic to $\mathcal{S}$. For each (c) $\in \mathrm{Cnt}_{m}$ and $\S \in$ Sem:

(TF9) (c) : $\mathcal{V}^{m} \longrightarrow \mathcal{V}$ is a total mapping such that: (TF10) $\S\left(C\left(\alpha_{0}, \ldots, \alpha_{m-1}\right)\right)=\left(C\left(\S\left(\alpha_{0}\right), \ldots, \S\left(\alpha_{m-1}\right)\right)\right.$. 
As advanced in the previous section, the latter two maxims are meant to embody a very specific, yet quite customary, version of the so-called (Fregean) Principle of Compositionality: The meaning of the whole is assumed to depend functionally on the meaning of its directly subordinated parts.

Any semantics conforming to the above 10 maxims is called a truth-tabular semantics (over $\mathcal{V}$ ), or a $\operatorname{Card}(\mathcal{V})$-valued truth-tabular semantics. Of course, a logic may easily have both a $\mathrm{K}$-valued and a $\lambda$-valued truth-tabular semantics, with $\kappa<\lambda$ : Just imagine for instance the situation in which $(\lambda-\kappa)$ new truthvalues are added to the former semantics that imitate precisely the behavior of other values that are already present. An excessive number of truth-values can also be present in other situations, where no pair of them are identifiable without prejudice to the underlying logic being characterized (an example of this phenomenon, typical of single-conclusion consequence relations, may be found in chapter 1 of [38]). To avoid that kind of ambiguity or redundancy, we call a $\operatorname{logic} \mathcal{L}$ genuinely $\mathrm{k}$-valued in case $\mathrm{k}=\operatorname{Min}(\{\operatorname{Card}(\mathcal{V}): \mathcal{L}$ has an adequate truth-tabular semantics over $\mathcal{V}\}$ ).

Remark 4 One might notice that this paper tries to avoid the use of the expression matrix semantics in favor of the present auspiciously less ambiguous expression truth-tabular semantics. The reason is that the former expression, coined and celebrated by the Polish school (cf. [22]), comes sometimes in slightly different realizations that do not always respect all the above maxims. For instance, when one talks about the intersection of arbitrary logics with a matrix semantics over a fixed language as defining a new logic with a matrix semantics in its own right, as in the case of the logic defined through a Lindenbaum bundle (recall Remark 3 and cf. [45, 29]), maxim (TF6), as we have seen, is in general disrespected. To avoid any risk of confusion, thus, I found it wiser to employ here the above novel and more specific terminology.

Example 5 Here are some standard examples of propositional logics characterized by truth-tabular semantics:

(i) Classical logic is genuinely 2-valued (any doubts?).

(ii) Łukasiewicz's logics $\mathrm{Ł}_{n}$, for $n \in \mathbb{N}$, are genuinely $n$-valued, while $\mathrm{E}_{\omega}$ is genuinely infinite-valued (cf. [14, 38]).

(iii) Intuitionistic logic is not genuinely finite-valued (cf. [19]), but it is infinite-valued (cf. [21]).

(iv) Most usual normal modal logics fail to be finite-valued (cf. [17]).

(v) Many paraconsistent logics and Logics of Formal Inconsistency fail to be finite-valued; several 3-valued (maximal) truth-functional samples of such logics, though, are known (cf. [12]).

Remark 6 How does one actually prove that a logic $\mathcal{L}$ has no genuine characterization in terms of finite-valued truth-tables? Suppose $\mathcal{L}$ is presented in 
proof-theoretical terms. Say that $\mathcal{L}$ has a sound semantics Sem induced by a set of truth-tables if such truth-tables validate all axioms and inference rules of $\mathcal{L}$, that is, if $\models$ Sem $\chi$ and $\Gamma \models$ sem $\varphi$ hold good for every axiom $\chi$ and every inference rule $\Gamma \Rightarrow \varphi$ of $\mathcal{L}$. If the converse is also the case, then Sem is said to provide an adequate semantics for $\mathcal{L}$.

Now, the usual procedure (cf. [19, 17, 12] for details and examples) that shows uncharacterizability of $\mathcal{L}$ by finite-valued truth-tables comes in two steps, for which a set of sentences $\Gamma \cup\{\varphi\}$ of $\mathcal{L}$ must be found with the following properties:

(i) $\Gamma \Rightarrow \varphi$ is valid in any sound finite-valued set of truth-tables for $\mathcal{L}$.

(The proof of this part is usually just a combinatorial exercise, and to that effect one might well make use, say, of the Pigeonhole Principle.)

(ii) $\Gamma \Rightarrow \varphi$ is not a consequence of the axioms and rules of $\mathcal{L}$.

(Now one has to find some sort of interpretation for $\mathcal{L}$ that does not validate $\Gamma \models$ $\varphi$. This model could be based, for instance, on a sound infinite-valued set of truth-tables for $\mathcal{L}$.)

Part (ii) of the above procedure, following [39], is traditional in the literature. The basic intuition behind it is usually attributed to independent proposals by Paul Bernays and Jan Łukasiewicz, published in the 1920s.

Remark 7 Classical propositional logic shares many useful properties with other finite-valued logics. Among these, perhaps compactness and decidability count as those with the most immediate computational interest. As a matter of fact, just like in the case of classical logic, any inference of a genuinely finitevalued logic can obviously be checked for validity, for instance, by way of a simple and well-defined (yet impractical) finitary truth-tabular procedure.

One can never insist too much on the discretionary character of the 10 above maxims, in the sense that they do not constitute anything like 'necessary requirements' for what we call logic. Besides, the maxims do not even provide the only possible approach to the notion of truth-functionality. Several less traditional extensions of the intuitive notion of truth-functionality have in fact been proposed in studies such as [36], [33], and many others. In that same direction, a particularly promising line of research has recently been opened with the proposal of the so-called 'non-deterministic multiple-valued structures' (cf. [3]).

Remark 8 A logic is said to be characterized by a non-deterministic truthtabular semantics in case it can be given an adequate semantics conforming to (TF1) to (TF8), plus the following two maxims. For each $(c) \in \mathrm{Cnt}_{m}$ and $\S \in$ Sem:

$\mathbf{N}$ (TF9) (c) : $\mathcal{V}^{m} \longrightarrow \operatorname{Pow}(\mathcal{V}) \backslash \varnothing$ is a total mapping such that:

$\mathbf{N}($ TF 10 $) \S\left(\subset\left(\alpha_{0}, \ldots, \alpha_{m-1}\right)\right) \in\left(C\left(\S\left(\alpha_{0}\right), \ldots, \S\left(\alpha_{m-1}\right)\right)\right.$.

According to the latter maxims, there might be a number of options for the interpretation of a given sentence. The set of valuations is still the set of homomorphisms from the algebra of the sentences into the algebra of truth-values, but 
now there is not necessarily a function that associates to each $m$-ary operator and each $m$-tuple of sentences a single truth-value depending on the truth-values of the latter sentences; there is, in general, a number of possible truth-values that can be associated to the sentence written with the former operator. By way of an example, given a unary 'possibility' operator $\diamond$ and a 2-valued set of truth-values $\{0,1\}$, one could well suppose that $\diamond p$ always assume the value 1 whenever $p$ is assigned the value 1 , but that the value of $\diamond p$ can be either 0 or 1 when $p$ is assigned the value 0 . So, we are sure that $p$ is possibly true when $p$ is in fact true, but we are unsure of its value otherwise ( $p$ might be only contingently false in a given state of affairs). Another interesting and opportune example would be that of a 0 -ary operator $\odot$ and a $\kappa$-valued set of truth-values $\{F, T\} \cup \mathcal{I}$, where $\mathcal{I}$ is a collection of 'intermediate values', such that $\odot$ is only allowed to assume one of the extreme values $F$ or $T$. The latter example does not seem easy to reconstruct in the scope of a deterministic semantics, where 0 -ary operators must be interpreted as assuming a single value throughout the semantics.

One attractive feature of non-deterministic semantics is that in the finitevalued case it still shares the standard computational properties of compactness and decidability, and at the same time it might still be said to respect some slightly more liberal version of the Principle of Compositionality. However, now it will be the case that finite-valued non-deterministic semantics can be shown to characterize both some logics that have only genuinely infinite-valued truth-tabular semantics (cf. [3] again) and also some logics that are non-truthfunctional (see the next section). On the other hand, it can also be shown that some logics are non-deterministically genuinely infinite-valued - yet they might still be perfectly decidable (cf. [2]). We will return to these specific issues in the final section of this paper.

Remark 9 Yet again, it is worth emphasizing that the canonical notion of entailment crystallized in maxim (TF3) is by no means unique. A prominent variant of it, frequently to be found for instance in the literature on manyvalued semantics for vague languages (cf., e.g., [24]), considers the case in which a partial order $\sqsubseteq$ is defined on the truth-values for which the greatest lower bounds (glb) exist, and set the ordered local entailment relation to be such that $\Gamma \models \sqsubseteq \frac{\sqsubseteq}{\S} \alpha$ iff $\operatorname{glb}(\S(\Gamma)) \sqsubseteq \S(\alpha)$. The underlying intuition here is that of preservation of truth-degree from premises to conclusion; to put it in a slogan, one might say that "degrees of truth are not lost from premises to conclusion" or that "conclusions are no less true than their premises". Now, on the one hand, the unordered notion of entailment that we have been calling 'canonical' is obviously a particular case of the ordered one, for which we design $v_{1} \sqsubseteq$ $v_{2}$ to fail only when $v_{1} \in \mathcal{D}$ and $v_{2} \in \mathcal{U}$. On the other hand, to simulate the ordered entailment using generalized matrices (recall Remark 3) one might define the family of all quasi-partitions $\langle\mathcal{D}, \mathcal{U}\rangle$ of $\mathcal{V}$ where the set of designated values $\mathcal{D}$ is a filter over $\mathcal{V}$ with respect to $\sqsubseteq$. As we commented before, it is not at all clear whether a generalized matrix such as this gives origin to a truth-tabular semantics complying to our original intuitive notion of truth- 
functionality. Thus, from this point on in our investigation, we will remain primarily with the unordered canonical notion of entailment, and try to conform closely to maxims (TF1)-(TF10).

The next section will show how our formal reconstruction of the intuitive notion of truth-functionality may be characterized from an abstract viewpoint. Several examples of logics that fail that characterization will then be exhibited, in more or less detail. Finally, the succeeding section will reinforce the importance of drawing a sharp distinction between the notion of truth-functionality as a property of a semantics and the same notion as a property of a logic (the present paper deals primarily with the latter, not the former). We will let the curtain drop soon after that, as we mention some directions for further generalization and investigation.

\section{Into the abstract}

Nobody should be afraid of abstract things and very abstract things, especially in mathematics; mathematics, precisely because it's mathematics, is abstract. More abstract a thing is [sic], it includes larger fields and so it is applicable on more concrete situations.

- Grigore C. Moisil (1906-1973).

We now tackle a different approach to the above matter. In this section we look at logic from an abstract viewpoint and see what is needed to characterize at that level the truth-functional behavior of a consequence relation. After a survey of some known results in Universal Logic, several examples of truth-functional and of non-truth-functional logics will be exhibited.

Let a propositional logic $\mathcal{L}$ be here described from an abstract viewpoint by a set of sentences $\mathcal{S}_{\mathcal{L}}$ and a (single-conclusion) consequence relation $\Vdash_{\mathcal{L}} \subseteq$ $\operatorname{Pow}\left(\mathcal{S}_{\mathcal{L}}\right) \times \mathcal{S}_{\mathcal{L}}$. As usual, we write a clause like $\left(\Gamma, \Delta \Vdash_{\mathcal{L}} \varphi\right)$ to indicate that the conclusion $\varphi \in \mathcal{S}_{\mathcal{L}}$ can be inferred from the set of premises $\Gamma \cup \Delta \subseteq \mathcal{S}_{\mathcal{L}}$. Subscripts and braces will be dropped whenever there is no risk of confusion. The above description defines an abstract structure that may or may not be subject to the following postulates, for $\Gamma \cup \Delta \cup\{\varphi\} \subseteq \mathcal{S}$ :

(AL1) $\Gamma, \varphi \Vdash \varphi$

(AL2) If $\Gamma, \Delta \Vdash \varphi$ and $(\forall \delta \in \Delta) \Gamma \Vdash \delta$, then $\Gamma \Vdash \varphi$

(AL3) If $\Gamma \Vdash \varphi$, then $\Gamma, \Delta \Vdash \varphi$

(dilution)

The consequence relations of most common logics respect the above postulates. Such logics are here called tarskian. Moreover, they quite often come equipped with some extra inner linguistic-related structure, together with a behavior that takes advantage of such structure:

(AL4) $\mathcal{S}$ has an algebraic character

(AL5) If $\Gamma \Vdash \varphi$, then $\Gamma^{\varepsilon} \Vdash \varphi^{\varepsilon}$, for any endomorphism $\varepsilon$ on $\mathcal{S}$

(substitutionality)

Logics respecting the latter postulates are here called substitutional. 
Remark 10 On what concerns the latter feature, the term 'structural', instead of 'substitutional', is more customary in the literature (at least since the publication of [22]). However, given the success in the latter years of the so-called substructural logics, I found it inadvisable to continue using this denomination. Indeed, the class of substructural logics is not characterized by the failure of (AL5), but by the proof-theoretical failure of any of a number of rules related to (AL3), to contraction and to permutation of the premises, considered as sequences of formulas.

A good question now is: In a substitutional context, when is a set of premises necessary for a given derivation? In particular, if one knows that, say, $\alpha, \beta \Vdash \gamma$ holds for a given logic, in which situation can we be sure that $\beta$ might be cancelled while the inference $\alpha \Vdash \gamma$ will still hold good? Say that a set of sentences $\Pi$ is $\mathcal{L}$-trivializing in case $(\forall \varphi \in \mathcal{S}) \Pi \Vdash \varphi$. Let $\operatorname{At}(\Pi)$ denote the set of atoms occurring along the sentences in $\Pi$, and say that $\Gamma$ and $\Delta$ are disconnected sets of sentences in case $\operatorname{At}(\Gamma) \cap \operatorname{At}(\Delta)=\varnothing$. Denote by $\left[\Pi_{i}\right]_{i<j}$ a list $\Pi_{0}, \Pi_{1}, \ldots, \Pi_{j-1}$. Consider then the following postulate as a possible answer to the above question:

(AL6) If $\left[\Gamma_{i}\right]_{i<j}, \Gamma \Vdash \varphi$, then $\Gamma \Vdash \varphi$,

(cancellation)

whenever $\Gamma \cup\{\varphi\},\left[\Gamma_{i}\right]_{i<j}$ are pairwise disconnected and no $\Gamma_{i}$ is $\mathcal{L}$-trivializing

The above postulate is intended to identify the situation in which there is no need to look at the $\Gamma_{i}$ 's to know whether $\varphi$ follows as a conclusion, but knowing instead the behavior of (the atoms of) $\Gamma$ should be enough. Logics respecting the latter postulate are here called cancelling.

So, here are two questions that arise naturally: (1) How do the above classes of abstract logics relate to the logics defined semantically in the previous section? (2) In particular, which of the above classes of abstract logics are guaranteed to have truth-functional semantics? The field of research that studies the connections between classes of logics realized abstractly and semantically is sometimes called Adequacy Theory (cf. [46]).

Fact 11 Let $\mathcal{L}=\langle\mathcal{S}, \Vdash\rangle$ be a logic. Then:

(A) $\mathcal{L}$ is tarskian if, and only if, it can be characterized by a semantics satisfying maxims (TF1), (TF2) and (TF3).

(B) A tarskian logic $\mathcal{L}$ is substitutional if, and only if, it can be characterized by a semantics satisfying also maxims (TF4) and (TF5).

(C) Let $\mathcal{L}$ be such that $\operatorname{Card}(\mathcal{S})=\operatorname{Card}(\mathrm{At})$ (as in the usual case of a denumerable set of atoms). A tarskian substitutional $\operatorname{logic} \mathcal{L}$ with this cardinality property is cancelling if, and only if, it can be characterized by a semantics satisfying also maxims (TF6) to (TF10), that is, iff it can be characterized by a truth-functional semantics. 
The result in part (B) is usually attributed to Lindenbaum and constitutes now a classic result in the 'theory of logical matrices' (cf. [23]). Its more general version, in part (A), is equally immediate to prove (cf. [6]), if we consider the global consequence relation defined by the Lindenbaum bundle (recall Remark 4). Less widely well-known, the result in part (C) was first proven in [44], using a somewhat different property than cancellation (called 'uniformity'). The simpler version formulated above, with the restriction on the cardinality of the set of atoms, comes from [38].

Remark 12 To a different notion of entailment, a different adequacy result may apply. Recall for instance the inherently trivalent notion of entailment introduced in Remark 2. It has been shown in [26] that a logic satisfies maxims (TF1), (TF2) and $(\mathrm{TF} 3)^{Q}$ if, and only if, its consequence relation respects postulate (AL3) plus the following alternative postulate:

$$
(\mathrm{AL} 2)^{Q} \text { If } \Gamma, \varphi \vDash \psi \text { and } \Gamma \vDash \varphi \text {, then } \Gamma \vDash \psi . \quad \text { (cautious cut) }
$$

Example 13 All of the following propositional logics respect postulates (AL1) to (AL5), but some of them fail (AL6):

(i) Classical logic respects cancellation. The same is also true for its negationless fragment, or for any other fragment containing only classical connectives.

(ii) Intuitionistic logic and all usual normal modal logics respect cancellation. They can all be given, thus, in the light of Fact 11(C), characteristic infinite-valued truth-functional semantics (recall the results about the inexistence of finite-valued characterizations, mentioned in Example 5(iii)(iv) and discussed in Remark 6).

(iii) Johánsson's minimal intuitionistic logic fails cancellation, as it has been remarked in [38]. It is instructive to recall (following [34]) how this logic is defined, and why it is not cancelling. Consider $I L^{+}$, the positive fragment of intuitionistic logic, as written for instance over the signature $\{\wedge, \vee, \rightarrow\}$ and governed by the usual hilbertian axioms and rules. Add now to this signature a 0-ary symbol I, but no further axioms. This is enough to define a new logic $h J$, the so-called minimal intuitionistic logic. From an algebraic point of view, $h J$ can be put in correspondence with a structure of the form $\langle A, \wedge, \vee, \rightarrow, I, 1\rangle$, called a $j$-algebra. In a $j$-algebra, $\langle A, \wedge, \vee\rangle$ is a distributive lattice, $a \rightarrow b$ denotes the relative pseudo-complement of $a$ with respect to $b, 1$ is its top element, and the botop I is interpreted as an arbitrary element of the carrier $A$. There are now two extreme things that can be done with a botop: It may be identified with the top element of the algebra, or instead with the bottom element of the algebra, when it exists. The logic resulting from the first choice, and the corresponding proof-theoretical addition of $I$ as a new axiom, is called $h J(T)$. The second choice may be accomplished by adding to $h J$ the schematic axiom $I \rightarrow \beta$. In this case, the resulting logic is called $h J(\perp)$, and the corresponding $j$-algebra 
is known as a Heyting algebra. Indeed, $h J(\perp)$, as the reader will have recognized, is just another name for the intuitionistic logic $(I L)$.

It is worth insisting that, by the mere addition of a new 0-ary connective symbol to the signature, $h J$ does indeed constitute a conservative extension of $I L^{+}$. The fact is better illustrated with the definition of a certain unary connective $\sim$, by setting $\sim \alpha \stackrel{\text { def }}{=} \alpha \rightarrow I$, for every $\alpha \in \mathcal{S}$. It is easy to check, then, using the properties of intuitionistic implication, that the following inferences / theorems are derivable in $h J$ :

$$
\begin{aligned}
& \alpha, \sim \alpha \vdash \sim \beta \\
& \vdash \alpha \rightarrow \sim \sim \alpha \\
& \vdash(\alpha \rightarrow \beta) \rightarrow((\alpha \rightarrow \sim \beta) \rightarrow \sim \alpha) \\
& \vdash(\alpha \rightarrow \sim \beta) \rightarrow(\beta \rightarrow \sim \alpha)
\end{aligned}
$$

These are typical theorems involving the intuitionistic negation. Furthermore, in $h J(\perp)$, one can also derive:

$$
\alpha, \sim \alpha \vdash \beta
$$

To obtain $C L^{+}$, the positive fragment of classical logic, one might add to $I L^{+}$as a new axiom the so-called Peirce's Law, $((\alpha \rightarrow \beta) \rightarrow \alpha) \rightarrow \alpha$. From the algebraic point of view, this results in letting $a \rightarrow b$ denote the relative complement of $a$ with respect to $b$. If one now adds a botop to the signature of $\mathrm{CL}^{+}$, one defines $h K$, the so-called 'logic of classical refutability' (thus called, in [15], following a 1958 unpublished manuscript by Kripke, in contrast to $h J$ 's 'simple refutability'). From the proof-theoretical point of view, this allows us to derive excluded middle, as conveyed by theorems such as:

$$
\begin{aligned}
& \vdash(\alpha \rightarrow \sim \alpha) \rightarrow \sim \alpha \\
& \vdash(\alpha \rightarrow \beta) \rightarrow((\sim \alpha \rightarrow \beta) \rightarrow \beta)
\end{aligned}
$$

As in the case of $h J$, one may next extend $h K$ into $h K(\top)$ or $h K(\perp)$. Of course, $h K(\perp)$ is just another name for classical logic $(C L)$, and the special class of Heyting algebras that corresponds to it determines the well-known class of Boolean algebras. In $C L$ one can also derive theorems such as:

$$
\begin{aligned}
& \vdash \sim \sim \alpha \rightarrow \alpha \\
& \vdash(\sim \alpha \rightarrow \beta) \rightarrow((\sim \alpha \rightarrow \sim \beta) \rightarrow \alpha) \\
& \vdash(\sim \alpha \rightarrow \beta) \rightarrow(\sim \beta \rightarrow \alpha)
\end{aligned}
$$

Recall now the postulate of cancellation, (AL6), and let $j=1, \Gamma_{0}=$ $\{p, \sim p\}, \Gamma=\varnothing$, and $\varphi=\sim q$, where $p$ and $q$ are any two distinct atoms of the language. Next, observe that in both $h J$ and $h K$ the inference $p, \sim p \vdash \sim q$ holds good, $\{p, \sim p\}$ is not trivializing, yet $\vdash \sim q$ does not hold. These logics fail, thus, (AL6), and so we may conclude that they are nontruth-functional. The same line of reasoning cannot be applied though 
to $h J(\top)$ and $h K(\top)$, as $\vdash \sim q$ is derivable in these logics. Similarly, it cannot be applied to $h J(\perp)$ and $h K(\perp)$, as $\{p, \sim p\}$ is a trivializing set of sentences in such logics.

A summary of the above constructions can be found in Fig. 4.

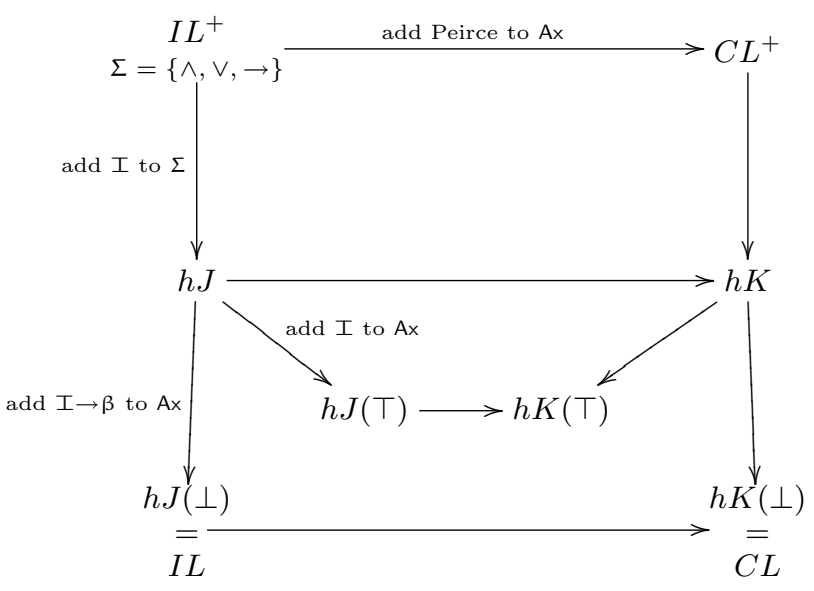

Figure 4: Johánsson and his family.

(iv) Lewis's modal logics $S 1, S 2$ and $S 3$, nowadays largely overlooked in the literature (they did not fully respect the usual necessitation rule of normal modal logics), all fail cancellation. This has also been observed in [38].

(v) All the main paraconsistent logics respect cancellation (take into account though Example 5(v)). There is a number of paraconsistent logics, however, that do fail the cancellation postulate. Recall that a logic $\mathcal{L}$ is paraconsistent if it has a symbol $\neg$ for negation such that $p, \neg p \vDash q$ does not hold good. This is intended to convey the idea that contradictions should not necessarily explode, that is, they should not allow, in general, for arbitrary sentences whatsoever to be derived from them. Now, it might still be the case that some 'partial explosion' is allowed by $\mathcal{L}$ (cf. [43]). Suppose there is some non-derivable non-atomic schematic sentence $\sigma$ such that $\operatorname{At}(\sigma)=q$, and suppose that $p, \neg p \vDash \sigma$ does hold good. Then $\mathcal{L}$ is said to be partially explosive (with respect to $\sigma$ ) (cf. [13]). Examples of partially explosive paraconsistent logics are provided by $h J$ and $h K$ in item (iv), above. Clearly, any such logic fails cancellation; thus, none of them can be truth-functional.

The next section will comment on what might happen when the notion of truth-functionality is ambiguously understood. The paper will finish by briefly entertaining some attractive generalizations of this notion. 


\section{Non-truth-functional logics versus non-truth-functional semantics}

Ideas do not have to be correct in order to be good; it's only necessary that, if they do fail, they do so in an interesting way.

-Robert Rosen (1934-1998).

The previous sections have recalled how truth-functional logics (as individuated by their associated consequence relations) may be characterized both semantically, in terms of genuinely finite-valued truth-tabular semantics, and abstractly, in terms of postulates regulating the properties of their underlying consequence relations. Now, a common confusion found in the literature involves the misidentification of (NT1) 'non-truth-functional logics' with (NT2) 'logics presented by way of a non-truth-functional semantics'. Indeed, while (NT1) makes reference to an objective property of a given consequence relation, (NT2) refers to a circumstantial mode in which a given logic happens to be semantically presented, at some given moment. As a matter of fact, a truth-functional logic can quite well be semantically presented by way of a non-truth-functional semantics conforming to maxims (TF1) to (TF8) but failing to be constituted of a set of homomorphisms between two similar algebras.

The above mentioned phenomenon may easily be illustrated if we consider, for instance, Lukasiewicz's well-known 3-valued logic $\mathrm{E}_{3}$, where $\mathrm{Cnt}_{1}=\{\sim\}$, $\mathrm{Cnt}_{2}=\{\rightarrow\}$, and $\mathrm{Cnt}_{n}=\varnothing$ for $n \notin\{1,2\}$, and where a genuinely finite-valued semantics $\operatorname{Sem}_{\S}$ is defined by setting the truth-values $\mathcal{D}=\{1\}, \mathcal{U}=\left\{\frac{1}{2}, 0\right\}$, and the interpretations $\sim: \mathcal{V} \longrightarrow \mathcal{V}$ and $\rightarrow: \mathcal{V} \times \mathcal{V} \rightarrow \mathcal{V}$ such that $\S(\sim \alpha)=1-\S(\alpha)$ and $\S(\alpha \rightarrow \beta)=\operatorname{Min}(1,1-\S(\alpha)+\S(\beta))$. Now, while this semantics is perfectly truth-functional, another — non-truth-functional - semantics may be provided to characterize the same entailment relation, as long as we abandon maxims (TF9) and (TF10), and consider instead a bivalent semantics $\mathrm{Sem}_{b}$ given by the collection of all total mappings $b: \mathcal{S} \longrightarrow\{T, F\}$ subject to the following restrictions:

$$
\begin{array}{llll}
\text { (b1) } & b(\alpha \rightarrow \beta)=T & \Leftrightarrow & b(\sim \alpha)=T \text { or } b(\beta)=T \\
& & & \text { or }(\text { both } b(\alpha)=F \text { and } b(\sim \beta)=F) \\
\text { (b2) } & b(\sim(\alpha \rightarrow \beta))=T & \Leftrightarrow & b(\alpha)=T \text { and } b(\sim \beta)=T \\
\text { (b3) } & b(\sim \sim \alpha)=T & \Leftrightarrow & b(\alpha)=T \\
\text { (b4) } & b(\sim \alpha)=T & \Rightarrow & b(\alpha)=F
\end{array}
$$

Observe how the above restrictions do not fully determine the truth-value of a complex sentence in terms of the atomic sentences that occur in it; in particular, for a given atom $p,(\mathrm{~b} 4)$ allows for either $b(\sim p)=T$ or $b(\sim p)=F$ when all we know is that $b(p)=F$. Nonetheless, if we consider the canonical notions of entailment associated to this bivalent semantics $\operatorname{Sem}_{b}$, where $\mathcal{D}_{b}=\{T\}$, it is straightforward to check that they coincide with the corresponding entailment relations associated to the previous genuinely 3 -valued semantics Sem $\$$. In fact, if we consider the total mapping $t: \mathcal{D} \cup \mathcal{U} \longrightarrow\{T, F\}$ such that $t(v)=T$ iff $v \in \mathcal{D}$, it is an easy exercise to check that $f \in \operatorname{Sem}_{b}$ iff there is some $g_{f} \in \mathrm{Sem}_{\S}$ 
such that $t \circ g_{f}=f$ and vice-versa, $g \in \operatorname{Sem}_{\S}$ iff there is some $f_{g} \in \mathrm{Sem}_{b}$ such that $f_{g}=t \circ g$.

Given a many-valued valuation $\S$, let's call the mapping to $\S$ the binary image of $\S$. The procedure that produces a 2 -valued version for any many-valued logic by taking the binary images of its original many-valued valuation mappings was suggested by Roman Suszko in the 1970s, in a number of papers starting with [41]. The corresponding general results may be found in [29], and an algorithm for constructively producing the set of restrictions on bivalent mappings corresponding to a given genuinely finite-valued semantics, for a class of sufficiently expressive logics that include most of the main many-valued logics from the literature, may be found in [6]. A report of implementation of that algorithm as an automated procedure for extracting sound and complete collections of tableau rules ready to use with a proof assistant may be found in [32], and a refinement of the above mentioned algorithm that allows for the extraction of collections of rules with the extra computationally convenient property of analyticity, ready for implementation in the form of an automated proof procedure, is presented at $[8]$.

It is never enough to insist that such a bivalent characterization of a genuinely $\kappa$-valued logic can never be truth-functional in case $\kappa>2$. The lesson to be learned from all this, of course, is that a truth-functional logic can perfectly well be presented in terms of a non-truth-functional semantics. From a conceptual point of view, there are at least two ways in which one may benefit from that lesson. The first one is the realization of the unsoundness of the attempts to classify logics according to the particular semantics in terms of which they are circumstantially presented, irrespective of their general abstract properties. This is the mistake committed in papers such as [35]. Indeed, from the purely semantical perspective, it might happen for instance that the consequence relation that happened to be associated to a logic $\mathcal{L}_{1}$, characterized by way, say, of a possible-worlds semantics, appears to be more 'intuitive' than the consequence relation that was associated to a $\operatorname{logic} \mathcal{L}_{2}$, characterized by way, say, of a many-valued semantics - or vice-versa. But nothing prevents, in principle, both $\mathcal{L}_{1}$ and $\mathcal{L}_{2}$ to be characterized at another moment in terms of other semantical presentations, more or less 'intuitive', informative and practically useful than the original ones. If one is lucky enough, it might even be possible to find an alternative semantic characterization that makes $\mathcal{L}_{2}$ look more 'intuitive' than $\mathcal{L}_{1}$. Historically, indeed, the multiple semantical characterization of the most successful logics in the literature has often been the case. What sense would it make thus to compare two logics and even argue in favor of the purported 'superiority' of one of them, on what concerns their use in the same domain of application, just by looking at the way such logics happened to be semantically characterized, at a given moment in time?

The second lesson concerns the need for greater care when using the term 'non-truth-functional' as applied to a logic. Indeed, as we have seen above, many logics such as intuitionistic logic, normal modal logics and many paraconsistent logics are genuinely infinite-valued. All the latter logics, however, can be characterized by way of appropriate bivalent non-truth-functional semantics, either 
obtained non-constructively just by invoking the corresponding binary images, or else obtained constructively by way of a number of restrictions on bivaluations that may be effectively checked and that provide a decision procedure for those logics. Yet again, as in the bivalent presentation of $\mathrm{七}_{3}$, we are still talking about truth-functional (in the above mentioned cases, infinite-valued) logics, even if they happen to be circumstantially presented by way of non-truth-functional semantics. Calling such logics 'non-truth-functional' is the conceptual mistake committed in [7] and [9].

\section{Going beyond}

If a little knowledge is dangerous, where is the man who has so much as to be out of danger?

-Thomas H. Huxley, Science \& Culture, 1881.

The previous sections have provided examples of logics with truth-tabular semantics (genuinely finite-valued or infinite-valued) and logics with no truthtabular semantics at all, and have pointed out how the corresponding semantically described classes of logics can be adequately characterized from an abstract point of view, in terms of single-conclusion consequence relations.

We have also illustrated in Remark 8 a possible generalization of the notion of truth-tabular semantics, called 'non-deterministic truth-tabular semantics'. As it turns out, many logics that are genuinely infinite-valued can in fact be characterized with the help of non-deterministic finite-valued semantics (cf. for instance $[3,2])$. In addition, it is interesting to see that even a non-truthfunctional logic such as $h K$ (recall Example 13(iii)) may be easily characterized in terms of non-deterministic 2 -valued semantics. Indeed, just consider the classical Card $(\{0,1\})$-valued interpretations of the connectives $\wedge, \vee$ and $\rightarrow$, and let the interpretation of $I$ as an operator, according to $\mathrm{N}(\mathrm{TF} 9)$, be given by the 0 -ary mapping $I=\{1,0\} \in \operatorname{Pow}(\{1,0\}) \backslash \varnothing$. This shows how the application field of 'non-deterministic multiple-valued structures' goes well beyond the usual field of multiple-valued structures. This example may be easily adapted for $h J$ using a combination of non-deterministic semantics and possible-worlds semantics, as in [1].

Just as it happens with truth-tabular semantics, some logics also turn out not to be characterizable by finite-valued non-deterministic truth-tabular semantics. Indeed, in [2] a certain class of paraconsistent logics, including the early da Costa's logic $C_{1}$ (cf. [16]), is shown to be genuinely infinite-valued even from a non-deterministic perspective. However, in $[28,10]$ we have shown how $C_{1}$ can be endowed with a possible-translations semantics based on 3 -valued 'factors', and the same is also true for a large number of other paraconsistent logics (cf. [31]). Possible-translations semantics, in fact, provide a further generalization of the notion of truth-functionality, and non-deterministic semantics form but a special case of them (cf. $[29,11])$. The underlying idea, in the case of many-valued factors, is to consider the controlled superposition of different simultaneous truth-functional scenarios, and interpret a formula in terms of all 
of its possible translations into such scenarios. This can be done by mantaining (TF10) as in the truth-functional case and modifying N(TF9) in such a way that there will be a new operator ${ }_{(}{ }_{k}$ for each deterministic choice of truth-table allowed by $\mathrm{N}(\mathrm{TF} 9)$ and $\mathrm{N}(\mathrm{TF} 10)$. In case the truth-functional factors chosen as scenarios have different sets of truth-values, one might also need to abandon maxim (TF6). In the example of $h K$, above, the maneuver corresponds to the choice of two possible translations for $I$, one such that $\operatorname{Tr}_{1}(I)=\perp$ and another one such that $\operatorname{Tr}_{2}(I)=\top$, where $\perp$ and $\top$ have their classical interpretations. If $\left\{\operatorname{Tr}_{k}: \mathcal{S} \longrightarrow \mathcal{S}_{k}\right\}_{k \in K}$ is the collection of admissible translations associated to a logic $\mathcal{L}$, and each $\mathcal{S}_{k}$ is the set of formulas of a truth-functional logic $\mathcal{L}_{k}$, we say that $\left\{\mathcal{L}_{k}\right\}_{k \in K}$ are the factors of $\mathcal{L}$ and define: $\Gamma \models_{T r} \alpha$ iff $\left(\Gamma \models_{\mathcal{L}_{k}} \alpha\right.$ for every $k \in K)$. A further advantage of possible-translations semantics, in terms of its capability to generalize (deterministic or non-deterministic) truthfunctional semantics, consists in the possibility of defining the collection $\mathrm{Tr}$ by positing arbitrary collections of restrictions over the admissible translations, further generalizing the format of $\mathrm{N}(\mathrm{TF} 9)$. In case $T r$ is recursively defined, each formula has a finite number of possible translations and the factor logics are finite-valued, the whole procedure is effective and decidability follows easily.

Though both non-deterministic semantics and possible-translations semantics appear to be very useful and appealing generalizations of the traditional notion of truth-functionality, their scope of application is not obvious. Their full abstract characterizations are still to be investigated, thus, following the line of exposition illustrated by the adequacy results that can be found in section 3 , specially if we recall the argument in section 4 about not trusting any circumstantial semantical characterization of a logic to give us objective information about its actual status as regards truth-functionality.

Last, another important line of investigation concerns the reformulation of the above mentioned adequacy theorems in order to deal with multipleconclusion logics. As a matter of fact, in [29] a survey of the multiple-conclusion versions of Fact 11(A) and (B) can be found. We are unaware, though, of a multiple-conclusion version of Fact $11(\mathrm{C})$ in the literature, to the best of our knowledge. On what concerns the associated notions of entailment, in (TF3) we have already stated the definitions in such a way as to accommodate the multiple-conclusion case - all you have to do to test whether $\Gamma \models_{x} \Delta$ is to replace $\Delta$ for $\{\alpha\}$ in the corresponding definitions. Semantical characterizations of multiple-conclusion logics are specially important if you consider their binary images and the fundamental categoricity result (cf. [20]) according to which each multiple-conclusion tarskian logic is characterized by a unique bivalent semantics (up to isomorphism). This is not the case for single-conclusion logics (or for non-bivalent semantics). For instance, classical logic with an extra trivial valuation (a valuation satisfying all formulas of its language) added to its usual truth-functional semantics would define exactly the same single-conclusion consequence relation, yet would appear to be 'paraconsistent' (in having a model for each and every contradiction). The same trick may be applied in fact to any other consistent logic (cf. [30]) — unless we choose to work in a multipleconclusion framework. 
Acknowledgements. The author acknowledges partial support by the CNPq research grant 304599/2008-5 and by the Fapesp Thematic Project ConsRel 2004/14107-2. Special thanks are also due to Carlos Caleiro for useful discussions in the final stage of this study, to two anonymous referees for their careful reading and appreciation, and to all the audiences who have endured the author's presentations of early outlines of the above ideas.

\section{References}

[1] Avron, Arnon, 'A nondeterministic view on nonclassical negations', Studia Logica, 80 (2005), 159-194.

[2] Avron, Arnon, 'Non-deterministic semantics for families of paraconsistent logics', in Béziau et al. [4], pp. 285-320.

[3] Avron, Arnon, and Iddo Lev, 'Non-deterministic multiple-valued structures', Journal of Logic and Computation, 15 (2005), 241-261.

[4] Béziau, Jean-Yves, Walter Carnielli, and Dov Gabbay, (eds.) Handbook of Paraconsistency, vol. 9 of Studies in Logic, College Publications, 2007.

[5] Caleiro, Carlos, Paula Gouveia, and Jaime Ramos, 'Hierarchical logical consequence', Preprint, SQIG - IT and IST - TU Lisbon, 1049-001 Lisboa, Portugal, 2007. Submitted for publication.

[6] Caleiro, Carlos, Walter Carnielli, Marcelo E. Coniglio, and JoÃo MARCOS, 'Two's company: "The humbug of many logical values", in J.-Y. Béziau, (ed.), Logica Universalis, Birkhäuser Verlag, Basel, Switzerland, 2005, pp. 169-189. Preprint available at:

http://wslc.math.ist.utl.pt/ftp/pub/CaleiroC/05-CCCM-dyadic.pdf.

[7] Caleiro, Carlos, Walter A. Carnielli, Marcelo E. Coniglio, Amílcar Sernadas, and Cristina Sernadas, 'Fibring non-truthfunctional logics: Completeness preservation', Journal of Logic, Language and Information, 12 (2003), 2, 183-211.

[8] Caleiro, Carlos, and João Marcos, 'Classic-like analytic tableaux for finite-valued logics', in H. Ono, M. Kanazawa, and R. de Queiroz, (eds.), Proceedings of the XVI Workshop on Logic, Language, Information and Computation (WoLLIC 2009), held in Tokyo, JP, June 2009, vol. 5514 of Lecture Notes in Artificial Intelligence, Springer, 2009, pp. 268-280. Preprint available at:

http://wslc.math.ist.utl.pt/ftp/pub/CaleiroC/09-CM-ClATab4FVL.pdf.

[9] Carnielli, W. A., M. E. Coniglio, D. Gabbay, P. Gouveia, and C. Sernadas, Analysis and Synthesis of Logics: How To Cut And Paste Reasoning Systems, vol. 35 of Applied Logic, Springer, 2008. 
[10] Carnielli, Walter A., 'Possible-translations semantics for paraconsistent logics', in D. Batens, C. Mortensen, G. Priest, and J. P. Van Bendegem, (eds.), Frontiers of Paraconsistent Logic, Proceedings of the I World Congress on Paraconsistency, held in Ghent, BE, July 29-August 3, 1997, Research Studies Press, Baldock, 2000, pp. 149-163.

[11] Carnielli, Walter A., and Marcelo E. Coniglio, 'Splitting logics', in S. Artemov, H. Barringer, A. Garcez, L. Lamb, and J. Woods, (eds.), We Will Show Them! Essays in Honour of Dov Gabbay, vol. 1, College Publications, 2005, pp. 389-414.

[12] Carnielli, Walter A., Marcelo E. Coniglio, and João Marcos, 'Logics of Formal Inconsistency', in D. Gabbay, and F. Guenthner, (eds.), Handbook of Philosophical Logic, vol. 14, 2nd edn., Kluwer, 2007, pp. 1-93. Preprint available at:

http://wslc.math.ist.utl.pt/ftp/pub/MarcosJ/03-CCM-lfi.pdf.

[13] Carnielli, Walter A., and João Marcos, 'A taxonomy of C-systems', in W. A. Carnielli, M. E. Coniglio, and I. M. L. D’Ottaviano, (eds.), Paraconsistency: The Logical Way to the Inconsistent, Proceedings of the II World Congress on Paraconsistency, held in Juquehy, BR, May 8-12, 2000, vol. 228 of Lecture Notes in Pure and Applied Mathematics, Marcel Dekker, 2002, pp. 1-94. Preprint available at:

http://wslc.math.ist.utl.pt/ftp/pub/MarcosJ/02-CM-taxonomy.pdf.

[14] Cignoli, Roberto L., Itala M. L. D’Ottaviano, and Daniele MundicI, Algebraic Foundations of Many-Valued Reasoning, vol. 7 of Trends in Logic, Kluwer, Dordrecht, 1999.

[15] Curry, Haskell B., Foundations of Mathematical Logic, McGraw-Hill, New York, 1963.

[16] Da Costa, Newton C. A., 'On the theory of inconsistent formal systems', Notre Dame Journal of Formal Logic, 11 (1974), 497-510.

[17] Dugundu, JAmes, 'Note on a property of matrices for Lewis and Langford's calculi of propositions', The Journal of Symbolic Logic, 5 (1940), $150-151$.

[18] Font, Josep M., and Ramon Jansana, A general algebraic semantics for sentential logics, vol. 7 of Lecture Notes in Logic, Springer-Verlag, Berlin, 1996.

[19] GöDEL, KuRT, 'Zum intuitionistischen Aussagenkalkül', Anzeiger Akademie der Wissenschaften Wien, mathematisch-naturwissenschaftliche Klasse, 69 (1932), 65-66. Reprinted in Ergebnisse eines mathematischen Kolloquiums, 4.

[20] Hardegree, Gary M., 'Completeness and super-valuations', Journal of Philosophical Logic, 34 (2005), 1, 81-95. 
[21] JaśKOWski, StanisŁaW, 'Recherches sur le systéme de la logique intuitionniste', in Actes du Congrès Internationale de Philosophie Scientifique, vol. 6 (Philosophie des mathèmatiques), Hermann, Paris, 1936, pp. 58-61.

[22] Łoś, Jerzy, and Roman Suszko, 'Remarks on sentential logics', Indagationes Mathematicae, 20 (1958), 177-183.

[23] Lukasiewicz, Jan, and Alfred TARski, 'Untersuchungen über den Aussagenkalküls', Comptes Rendus des séances de la Société des Sciences et des Lettres de Varsovie, 23 (1930), 30-50.

[24] Machina, Kenton F., 'Truth, belief and vagueness', Journal of Philosophical Logic, 5 (1976), 1, 47-78. Reprinted in R. Keefe and P. Smith (editors), Vagueness: A Reader, MIT Press, 1997.

[25] Makinson, David, 'Bridges between classical and nonmonotonic logic', Logic Journal of the IGPL, 11 (2003), 1, 69-96.

[26] Malinowski, Grzegorz, 'Q-consequence operation', Reports on Mathematical Logic, 24 (1990), 49-59.

[27] Malinowski, Grzegorz, 'Inferential many-valuedness', in J. Woleński, (ed.), Philosophical Logic in Poland, Kluwer, Dordrecht, 1994, pp. 75-84.

[28] Marcos, João, Possible-Translations Semantics (in Portuguese), Master's thesis, State University of Campinas, BR, 1999.

http://libdigi . unicamp.br/document/?code=vtls000224326.

[29] Marcos, Jỗo, 'Possible-translations semantics', in W. A. Carnielli, F. M. Dionísio, and P. Mateus, (eds.), Proceedings of the Workshop on Combination of Logics: Theory and applications (CombLog'04), held in Lisbon, PT, July 28-30, 2004, Departamento de Matemática, Instituto Superior Técnico, 1049-001 Lisbon, PT, 2004, pp. 119-128. July 28-30, 2004, Lisbon, PT. Extended version available at:

http://wslc.math.ist.utl.pt/ftp/pub/MarcosJ/04-M-pts.pdf.

[30] Marcos, JoÃo, 'Ineffable inconsistencies', in Béziau et al. [4], pp. 301311. Preprint available at:

http://wslc.math.ist.utl.pt/ftp/pub/MarcosJ/04-M-ii.pdf.

[31] Marcos, JoÃo, 'Possible-translations semantics for some weak classicallybased paraconsistent logics', Journal of Applied Non-Classical Logics, 18 (2008), 1, 7-28. Preprint available at:

http://wslc.math.ist.utl.pt/ftp/pub/MarcosJ/04-M-PTS4swcbPL.pdf.

[32] Marcos, JoÃo, and Dalmo Mendonça, 'Towards fully automated axiom extraction for finite-valued logics', in W. Carnielli, M. E. Coniglio, and I. M. L. D'Ottaviano, (eds.), The Many Sides of Logic, Studies in Logic, College Publications, London, 2009. Preprint available at:

http://wslc.math.ist.utl.pt/ftp/pub/MarcosJ/08-MM-towards.pdf. 
[33] Massey, Gerald J., 'The theory of truth tabular connectives, both truth functional and modal', The Journal of Symbolic Logic, 31 (1966), 593-608.

[34] Odintsov, Sergei P., 'On the structure of paraconsistent extensions of Johansson's logic', Journal of Applied Logic, 3 (2005), 1, 43-65.

[35] Priest, Graham, 'Paraconsistent logic', in D. M. Gabbay, and F. Guenthner, (eds.), Handbook of Philosophical Logic, vol. 6, 2nd edn., Kluwer, Dordrecht, 2002, pp. 259-358.

[36] Rescher, Nicholas, 'Quasi-truth-functional systems of propositional logic', The Journal of Symbolic Logic, 27 (1962), 1-10.

[37] Schurz, Gerhard, 'Logic, matter of form, and closure under substitution', in M. Bilkova, and L. Behounek, (eds.), The Logica Yearbook 2004, Filosofia, 2005, pp. 33-46.

[38] Shoesmith, D. J., and Timothy J. Smiley, 'Deducibility and manyvaluedness', The Journal of Symbolic Logic, 36 (1971), 4, 610-622.

[39] Smiley, Timothy, 'The independence of connectives', The Journal of Symbolic Logic, 27 (1962), 4, 426-436.

[40] Smiley, Timothy, 'Rejection', Analysis (Oxford), 56 (1996), 1, 1-9.

[41] Suszko, Roman, 'Abolition of the Fregean Axiom', in R. Parikh, (ed.), Logic Colloquium: Symposium on Logic held at Boston, 1972-73, vol. 453 of Lecture Notes in Mathematics, Springer-Verlag, Berlin, 1972, pp. 169-239.

[42] Tarski, Alfred, 'On the concept of following logically', History and Philosophy of Logic, 23 (2002), 155-196. Original from 1936.

[43] Urbas, Igor, 'Paraconsistency', Studies in Soviet Thought, 39 (1990), $343-354$.

[44] WóJCiCKI, RYSZARD, 'Logical matrices strongly adequate for structural sentential calculi', Bulletin de l'Academie Polonaise des Sciences, Série des Sciences Mathématiques, Astronomiques et Physiques, 17 (1969), 333-335.

[45] Wójcicki, RYszard, Theory of Logical Calculi, Kluwer, Dordrecht, 1988.

[46] Zygmunt, Jan, An Essay in Matrix Semantics for Consequence Relations, Wydawnictwo Uniwersytetu Wrocławskiego, Wrocław, 1984. 